cyanopindolol bound to purified $\beta_{1}$-AR to directly monitor ligand removal by dialysis, performed on the sample used to create the crystals, could have provided convincing evidence that all the cyanopindolol was actually removed and was therefore absent in the crystals produced.

The $R$ factors that we report are markedly higher than those reported by Huang et al. ( $R_{\text {work }} 30.99 \%$ and $R_{\text {free }} 35.46 \%$ ). There are two possible explanations for this: the first is differences in modeling the solvent contribution in PHENIX ${ }^{7}$ and REFMAC5; the second is the unusual distribution of atomic $B$ factors in the PDB 4GPO coordinate file, in which there are large differences in $B$ factors for bonded side chain atoms, including $B$ factors both increasing and decreasing along the length of the side chain (e.g., Met36 in chain A has $B$ factors of $75,30,24,118$ and $128 \AA^{2}$ for $\mathrm{CA}, \mathrm{CB}, \mathrm{CG}, \mathrm{SD}$ and $\mathrm{CE}$ atoms, respectively). This variation does not seem to be physically reasonable and may represent overfitting of the data. It would have been useful to carry out refinement against the original data rather than the anisotropically truncated and sharpened data that were deposited; however the original data were not made available to us. The $R$ factors for our final model are within the expected range for a structure refined at this resolution.

A consequence of the presence of cyanopindolol in the PDB 4GPO crystal structure is that the dimensions of the ligand-binding pocket need to be reassessed. Specifically, the contraction of the ligand-binding pocket reported by Huang et al. is no longer feasible, owing to steric clashes between the ligand and the side chains on helix 5 . The ligand-binding pocket in the structure that we have refined from the same data exhibits dimensions identical to those in the original cyanopindolol-bound structure (PDB 2VT4).

The discrepancies that we have described in the PDB 4GPO structure are in the ligandbinding pocket. However, the interesting packing of $\beta_{1}$-AR monomers within the crystal to form parallel dimers, which could be of physiological relevance, was unaffected upon rerefinement of the data.
Note: Any Supplementary Information and Source Data files are available in the online version of the paper (doi:10.1038/nsmb.3130).

ACKNOWLEDGMENTS

This work was funded by core Medical Research Council funding to C.G.T. (MRC U105197215) and A.G.W.L. (MRC U105184325).

\section{COMPETING FINANCIAL INTERESTS}

The authors declare no competing financial interests.

\section{Andrew G W Leslie, Tony Warne \& Christopher G Tate}

Medical Research Council (MRC) Laboratory of Molecular Biology, Cambridge, UK.

e-mail:cgt@mrc-lmb.cam.ac.uk

1. Huang, J., Chen, S., Zhang, J.J. \& Huang, X.-Y. Nat. Struct. Mol. Biol. 20, 419-425 (2013).

2. McCoy, A.J. et al. J. Appl. Crystallogr. 40, 658-674 (2007).

3. Warne, T. et al. Nature 454, 486-491 (2008)

4. Murshudov, G.N., Vagin, A.A. \& Dodson, E.J. Acta Crystallogr. D Biol. Crystallogr. 53, 240-255 (1997).

5. Nicholls, R.A., Long, F. \& Murshudov, G.N. Acta Crystallogr. D Biol. Crystallogr. 68, 404-417 (2012).

6. Baker, J.G. PLOS ONE 5, e15487 (2010).

7. Adams, P.D. et al. Acta Crystallogr. D Biol. Crystallogr. 66, 213-221 (2010).

\section{Huang et al. reply:}

In response to the points raised by Leslie, Warne and Tate ${ }^{1}$, we reexamined the structural model that we deposited in the Protein Data Bank (PDB 4GPO) and obtained similar results to those that we reported previously ${ }^{2}$; i.e., we could observe some small extra density but no ligands. In addition, we asked another protein crystallography group to check the deposited data set, and they were also unable to find ligands in our structure (Jean Jakoncic (Brookhaven National Laboratory), personal communication).

We then checked the electron density maps of our structure with other web servers. On the PDB_REDO web server, the maps from both the 'conservatively optimized structure' and the 'fully optimized structure' did not show extra density for a ligand in either chain A or chain B (http://www.cmbi.ru.nl/pdb_redo/gp/4gpo/ index.html). However, on the Electron Density Server (EDS), the map showed the presence of extra electron density in both chains. We do not know why the map on the EDS web server shows the presence of this extra density.

The presence or absence of cyanopindolol in our structural model was carefully discussed in the paper. From our radioligand binding studies, the $\beta_{1}$-adrenergic receptor $\left(\beta_{1}\right.$-AR) protein sample used for the crystallization study yielded the same $K_{\mathrm{d}}$ value for dihydroalprenolol as that in cells expressing wild-type $\beta_{1}$-ARs. This indicated that most $\beta 1$-ARs in the protein sample were not bound with cyanopindolol; otherwise, we would have observed a rightward shift in the competition curve. In addition, at $\mathrm{pH} 4$, the ligand binding was decreased by approximately ten-fold compared to that at $\mathrm{pH}$ 7.7. From our mass spectrometry analysis, we estimated that, if cyanopindolol were present, $<1 \%$ of receptors would be bound with cyanopindolol. One could argue that only cyanopindolol-bound $\beta 1$-ARs were stable; however, in our hands, purified $\beta 1-\mathrm{AR}(\mathrm{m} 23)$ proteins were stable at $\mathrm{pH} 4$ for up to 6 months.

\section{COMPETING FINANCIAL INTERESTS}

The authors declare no competing financial interests.

\section{Jianyun Huang, Shuai Chen, J Jillian Zhang \& Xin-Yun Huang}

Department of Physiology and Biophysics, Cornell University Weill Medical College, New York, New York, USA.

e-mail:xyhuang@med.cornell.edu

1. Leslie, A.G.W., Warne, T. \& Tate, C.G. Nat. Struct. Mol Biol. 22, 941-942 (2015)

2. Huang, J., Chen, S., Zhang, J.J. \& Huang, X.-Y. Nat Struct. Mol. Biol. 20, 419-425 (2013). 